\title{
Embedded Fibre Bragg Grating Array Sensors in Aluminium Alloy Matrix by Ultrasonic Consolidation
}

\author{
Chengbo Mou ${ }^{\text {a*}}$, Pouneh Saffari ${ }^{\text {a }}$, Dezhi Li ${ }^{\mathrm{b}}$, Kaiming Zhou ${ }^{\mathrm{a}}$, Lin Zhang ${ }^{\mathrm{a}}$, Rupert Soar ${ }^{\mathrm{b}}$, Ian \\ Bennion $^{\mathrm{a}}$ \\ a Photonics Research Group, School of Engineering \& Applied Science, Aston University, \\ Birmingham, UK, B4 7ET; \\ ${ }^{b}$ Rapid Manufacturing Research Group, Loughborough University, Loughborough, UK, LE11 3TU
}

\begin{abstract}
ABSTRCT
Fibre Bragg Grating (FBG) array sensors have been successfully embedded in aluminium alloy matrix by ultrasonic consolidation (UC) technique. The temperature and loading responses of the embedded FBG arrays have been systematically characterised. The embedded grating sensors exhibit an average temperature sensitivity of $\sim 36 \mathrm{pm} /{ }^{\circ} \mathrm{C}$, which is three times higher than that of normal FBGs, and a loading responsivity of $\sim 0.1 \mathrm{~nm} / \mathrm{kg}$ within the dynamic range from $0 \mathrm{~kg}$ to $3 \mathrm{~kg}$. This initial experiment clearly demonstrates that FBG array sensors can be embedded in metal matrix together with other passive and active fibres to fabricate smart materials to monitor the operation and health of engineering structures.
\end{abstract}

Keywords: Fibre Bragg Grating, Optical Fibre Sensor, Smart Structure, Ultrasonic Consolidation

\section{INTRODUCTION}

In-fibre Bragg gratings have been widely implemented as embeddable smart sensors for monitoring strain, temperature, bending, loading and cracking in numerous engineering structures ${ }^{1}$. FBG sensors have a range of application advantages over the traditional electric sensors. As tens of gratings of different wavelengths can be fabricated in a single length fibre, a structure embedded with wavelength multiplexed FBG fibres can possess self-sensing functionality to monitor quasidistributed parameters in real time. However, so far FBG sensors have been embedded only in glass and carbon fibre reinforced composite materials and structures and no work reported in metal matrix composites through solid state process. Ultrasonic consolidation (UC) is a solid-state rapid manufacturing process which can be used to make parts layer by layer and finish final profile by contour milling ${ }^{2}$. Such layer-consolidation formed metal structures may be embedded with FBG array sensors to make smart structures with self-monitoring and sensing capability.

In this paper, we report for the first time the embedding of FBG array sensors in aluminium alloy matrix composites by $\mathrm{UC}$ technique and the temperature and loading responses of these embedded samples.

\section{EMBEDDED FBG SENSOR ARRAYS}

\subsection{Fabrication of Fibre Bragg Gratings}

The FBG array sensors were UV-inscribed in single mode standard telecom (Corning SMF-28) fibres using a 244nm UV light from a CW frequency doubled $\mathrm{Ar}^{+}$laser and the scanning phase mask technique. In order to increase the photosensitivity of the fibre, the SMF-28 fibres were $\mathrm{H}_{2}$-loaded under 150 bar at $80^{\circ}$ for 48 hours prior to the UVinscription. The integrated phase mask used in the experiment has three $1 \mathrm{~cm}$-long mask patterns with different pitch sizes along one dimension separated by $1 \mathrm{~cm}$. Thus, using this mask three FBGs of different wavelengths can be written

\footnotetext{
*Email: mouc@aston.ac.uk
}

19th International Conference on Optical Fibre Sensors, edited by David Sampson, Stephen Collins, Kyunghwan Oh, Ryozo Yamauchi, Proc. of SPIE Vol. 7004, 70044B, (2008) 0277-786X/08/\$18 doi: 10.1117/12.786065 
in a single fibre in one scan. In order to accommodate the designed length of the metal matrix samples, $60 \mathrm{~mm}$ middle section of the host fibre samples were chemically stripped off for grating array inscription. Each grating length is about $8 \mathrm{~mm}$ and the space between two adjacent gratings is about $10 \mathrm{~mm}$. After the UV-inscription, all FBG arrays were annealed at $80^{\circ} \mathrm{C}$ for 24 hours to stabilise their property.

\subsection{Sample Preparation by Ultrasonic Consolidation}

Fig. 1 shows the schematic diagram of the ultrasonic consolidation system used to embed FBG array fibres in metal matrix composite samples. Two types of Aluminium Alloy - Al 3003 H18 (which is fully strain hardened) and $3003 \mathrm{O}$ (which is annealed and soft) - with a thickness of $100 \mu \mathrm{m}$ were used to form the embedding structure. Due to the large plastic flows may occur around the fibre during the consolidation process and relatively fragile property of the fibre, SMF-28 fibres embedded between two $100 \mu \mathrm{m}$ metal foils could be easily damaged by the sonotrode ${ }^{3}$. Therefore, two foils were monolithically pre-bonded by the UC machine to form a layer with the thickness of $200 \mu \mathrm{m}$. After pre-bonding, the FBG array fibre was placed between the two pre-bonded layers; this sandwiched structure was clamped at one end with the other end free for consolidation. In order to keep the balance and not to use too many FBG array fibres, two SiC fibres were placed on each side of the FBG array fibre between the two pre-bonded metal foils.

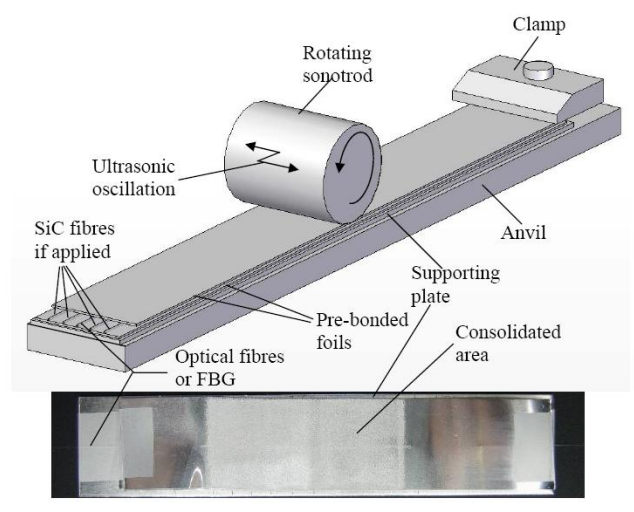

Figure.1. Schematic diagram of embedding FBG by ultrasonic consolidation.

As shown in Fig. 1, the sonotrode was laid down to contact with the sample where pressure is applied and ultrasonic oscillation takes place. After the sonotorde travelled from the clamped end to the free end, the FBG array fibre with four $\mathrm{SiC}$ fibres were embedded in the metal matrix composite in solid state. The matrix arrangement was plate/H18/O/FBG\&SiC/O/H18. For pre-bonding, the pressure was $114 \mathrm{MPa}$ and the amplitude of ultrasonic oscillation was $6.5 \mu \mathrm{m}$ and consolidation speed was $34.5 \mathrm{~mm} / \mathrm{s}$; for embedding, the pressure was increased to $155.8 \mathrm{MPa}$ and the amplitude of ultrasonic oscillation was $10.4 \mu \mathrm{m}$ and the transverse speed was kept the same as in pre-bonding process. Five FBG array embedded samples were produced and all the FBGs were survived from the UC embedding process.

\section{CHARACTERISATION OF THE EMBEDDED FIBRE SENSORS}

\subsection{FBG array characterisation}

Characterisation of FBG sensor arrays was carried out in their freeform and in embedded samples. The transmission spectra of the grating arrays were measured employing a broadband light source and an optical spectrum analyser, and the schematic diagram of the system is depicted in Fig.2a. The solid line in Fig. $2 b$ is the transmission spectrum of one of the UV-inscribed FBG arrays measured in its freeform, showing three Bragg peaks of $\sim 20 \mathrm{~dB}$ strength situated around $1542.8 \mathrm{~nm}, 1547.24 \mathrm{~nm}$ and $1551.84 \mathrm{~nm}$, respectively. The dotted trace in Fig. $2 \mathrm{~b}$ gives the measured transmission spectrum of the same FBG array after being embedded in the Al matrix composite. 
FBG Sample

\begin{tabular}{|l|l|}
\hline BBS & IIIIIII \\
\cline { 3 - 3 }
\end{tabular}

(a)

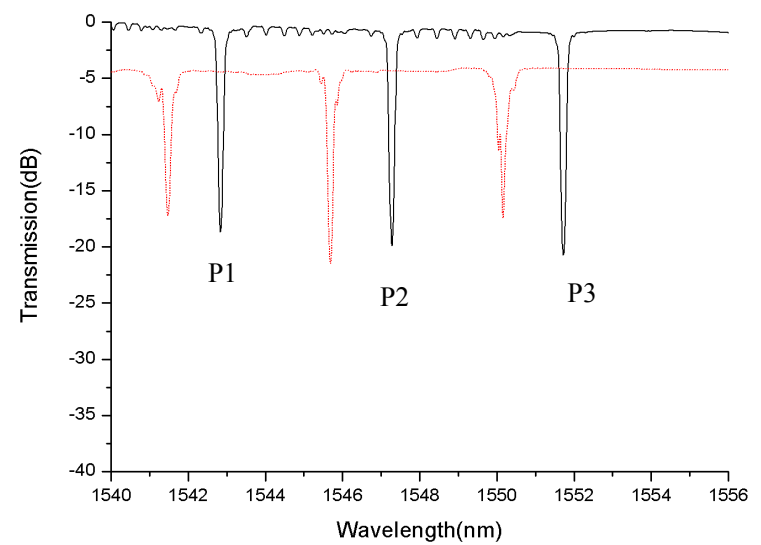

(b)

Figure.2. (a) Schematic diagram of the FBG array characterisation system; (b) measured transmission spectra of one of the FBG arrays before (solid line) and after (dotted line) embedding.

As shown clearly in Fig. 2b, we can see that the Bragg peaks shifted towards the shorter wavelength side after being embedded in the Al matrix composite. This was the most uniformly embedded sample as all three peaks have a similar blue-shift, about $2 \mathrm{~nm}$, and the grating strengths are slightly reduced and also no significant change is showing on the peak shapes. The other four embedded samples have shown different degrees of non-uniformity. The blue-shifts in these four samples were slightly different for the three Bragg peaks and some peaks showed broadening and splitting effects. Given that UC is a low temperature process, the blue shift of the Bragg resonances can only be the result of the mechanical compression induced by the UC to the embedded fibre.

\subsection{Characterisation of thermal responsivity}

The embedded FBG array samples were investigated for their thermal responsivity. Each sample was placed on an electrically controlled heater, as shown in Fig. 3a, and its spectrum were measured for temperature elevated from $0^{\circ}$ to $80^{\circ}$ with an increment of $5^{\circ}$. Fig. $3 \mathrm{~b}$ plots the wavelength shifts for the three Bragg peaks of the best embedded sample against temperature. The three peaks exhibit a near-linear and similar temperature response. The best fits to the three linear plots in Fig. $3 \mathrm{~b}$ give an average thermal responsivity of $\sim 36 \mathrm{pm} /{ }^{\circ} \mathrm{C}$. This value is more than three times of the typical value of normal FBGs in this wavelength range ${ }^{4}$. The increased thermal sensitivity is simply caused by the combination effect on thermal expansion of aluminium and silica glass materials.

Fibre Embedded Sample

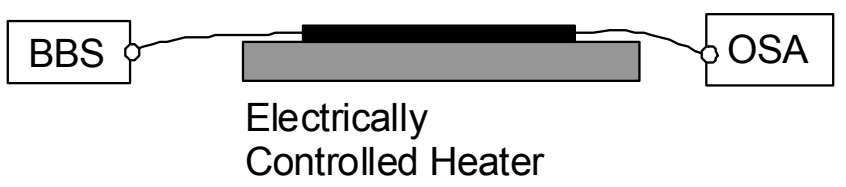

(a)

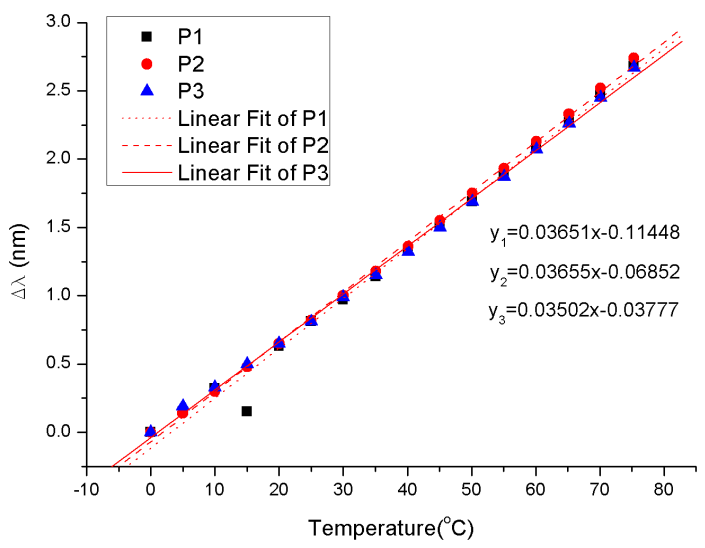

(b)

Figure.3. (a) Schematic diagram of the temperature sensing set-up; (b) thermal responses of the three Bragg peaks of the measured sample. 


\subsection{Investigation on loading response}

The embedded FBG array sample was subjected to loading experiment using a set-up shown in Fig. 4a. The weights from $0 \mathrm{~kg}$ to $9 \mathrm{~kg}$ were gradually loaded on the top of the embedded FBG array sample. Fig. $4 \mathrm{~b}$ depicts the wavelength shifts of the three peaks of the best sample against the weight. We can clearly see from Fig. $4 \mathrm{~b}$ there are two response regions corresponding to low and high loading range. In the low weight range, the Bragg peaks respond to the loading linearly with a relatively fast red-shift rate, around $0.1 \mathrm{~nm} / \mathrm{kg}$. When the applied load exceeds the threshold, the Bragg peaks shift at a much slower rate, in fact, their response to the loading is almost saturated. Unlike the thermal response, the three Bragg peaks exhibit non-uniform loading responses. P1 and P2 have a similar threshold load close to $3 \mathrm{~kg}$ whereas P3 has a much higher threshold at around $5 \mathrm{~kg}$. We also can see from fig. 4(b) that under the threshold load, P1 and $\mathrm{P} 3$ show a similar loading responsivity, $\sim 0.1 \mathrm{~nm} / \mathrm{kg}$, but $\mathrm{P} 2$ has a higher one, $\sim 0.2 \mathrm{~nm} / \mathrm{kg}$.

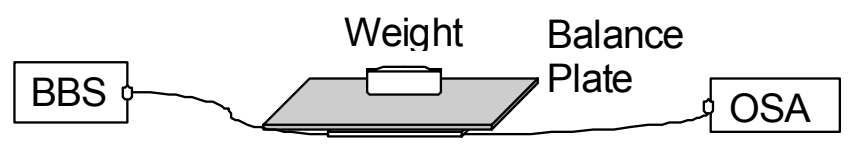

Fibre embedded (a)

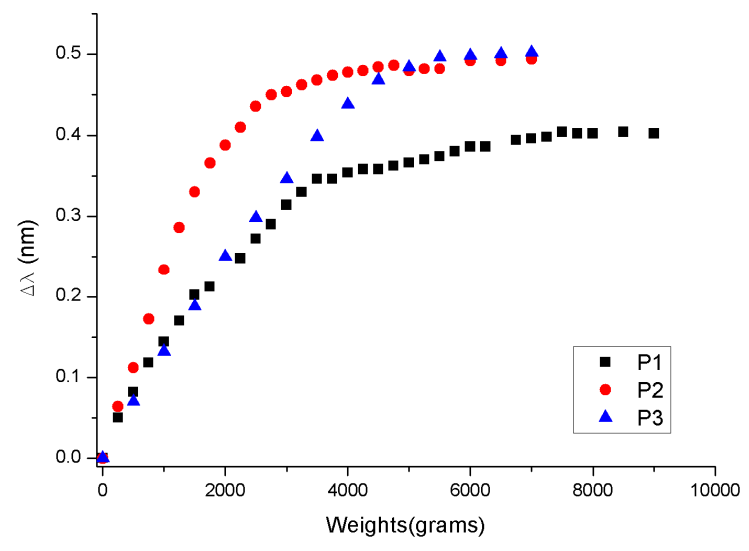

(b)

Figure.4. (a) Schematic configuration for loading experiment, (b) wavelength shift response to loading for Peak 1 (P1), Peak 2 (P2), and Peak 3 (P3).

\section{CONCLUSIONS}

In summary, for the first time we have embedded FBG array sensors in metal matrix composite by ultrasonic consolidation technique. The embedded samples have been investigated for their thermal and loading responses and the results have clearly demonstrated that these FBG embedded metal matrix samples possess self-sensing capability. We envisage that metal matrix based engineering tools and structures made by advanced solid-state rapid manufacturing technique UC may be embedded with FBG sensor arrays becoming smart structures, which would be beneficial to a range of applications.

\section{REFERENCE}

1. E.Udd, Fiber Optic Smart Structures, Wiley (Interscience), New York,1995

2. White, D.R, "Ultrasonic Consolidation of Aluminium Tooling," Advanced Materials \& Processes 161(1), 64-65 (2003).

3. Kong, C.Y and R.C.Soar, "Fabrication of metal-matrix composites and adaptive composites using ultrasonic consolidation process," Materials Science and Engineering a-Structural Materials Properties Microstructure and processing 412(1-2), 12-18 (2005)

4. L Zhang, W Zhang, I Bennion, "In-fibre grating optic sensors", Fibre Optic Sensors, ed. F T S Yu \& S Yin, (123181),Marcel Dekker, New York, 2002 